\title{
Arrhythmia Induced Cardiomyopathy: What are Predictors of Myocardial Recovery?
}

\author{
Acile Nahlawi ${ }^{1}$ and Marwan Refaat ${ }^{1}$ \\ ${ }^{1}$ American University of Beirut Medical Center
}

February 19, 2021

\section{Arrhythmia Induced Cardiomyopathy: What are Predictors of Myocardial Recovery?}

Acile Nahlawi BS, Marwan M. Refaat MD

Department of Internal Medicine, Division of Cardiology, American University of Beirut Medical Center, Beirut, Lebanon

Running Title: AIC and Predictors of Myocardial Recovery

Disclosures: None

Funding: None

Keywords: Cardiac Arrhythmias, Cardiovascular Diseases, Heart Diseases, Congestive Heart Failure, Cardiomyopathy

Words: 958 (excluding references)

Correspondence:

Marwan M. Refaat, MD, FACC, FAHA, FHRS, FASE, FESC, FACP, FRCP

Associate Professor of Medicine

Director, Cardiovascular Fellowship Program

Department of Internal Medicine, Cardiovascular Medicine/Cardiac Electrophysiology

Department of Biochemistry and Molecular Genetics

American University of Beirut Faculty of Medicine and Medical Center

PO Box 11-0236, Riad El-Solh 1107 2020- Beirut, Lebanon

Fax: +961-1-370814

Clinic: $+961-1-759616$ or $+961-1-355500$ or $+961-1-350000 /+961-1-374374$ Extension 5800

Office: +961-1-350000/+961-1-374374 Extension 5353 or Extension 5366 (Direct)

Email: mr48@aub.edu.lb

Cardiomyopathies cause a significant public health burden and improvement in sudden cardiac death risk stratification helped in decreasing mortality by improved pharmacotherapy as well as device implantations including implantable cardiac defibrillators and cardiac resynchronization therapy [1-4]. Arrhythmia induced cardiomyopathy (AIC) is a major cause of non-ischemic cardiomyopathy and heart failure (HF) worldwide [5]. It is characterized by an impairment of left ventricular systolic function secondary to high heart rate 
(tachycardia-induced), asynchrony (frequent premature ventricular contractions-induced or right ventricular pacing-induced) or an irregular rhythm (such as atrial fibrillation-induced) that serves as the trigger of AIC and this is mediated by calcium mishandling. The distinctive feature of AIC is the substantial improvement in left ventricular systolic function following arrhythmia suppression or elimination [5]. Atrial Fibrillation (AF) is concomitantly present with and potentially the cause of 10 to $50 \%$ of $\mathrm{HF}$ cases [6]. AIC is an important, commonly encountered and potentially reversible entity that is often under-recognized. The exact incidence and prevalence of AIC remains poorly defined in the literature [7]. In some studies, it was present in as high as $50 \%$ of patients with AF undergoing ablation, while it was reported to be present in $10 \%$ of patients with focal atrial tachycardia undergoing ablation [8]. In addition, very little attention, if any, is given to AIC in major trials on $\mathrm{AF}$ and $\mathrm{HF}$, despite its significant implications on morbidity and mortality and the promising benefits of treatment [7]. Many aspects of AIC are yet to be understood. In fact, few studies limited by small sample size constitute our main source of knowledge on extent and predictors of ventricular recovery after treatment initiation in patients with AIC $[9,10]$.

In their multicenter retrospective study, Gopinathannair et al. aimed to assess the degree of recovery of the left ventricular systolic function after suppression/elimination of the underlying arrythmia and to evaluate factors influencing this response such as baseline patient and arrhythmia characteristics. The study sample comprised 243 patients from 3 different institutions whose charts were reviewed retrospectively (no recruitment timeframe was indicated). The patient characteristics studied included baseline left ventricular ejection fraction (LVEF), presence of structural heart disease (SHD) [ defined as significant coronary artery disease, prior myocardial infarction, hemodynamically significant valvular heart disease, or other structural cardiomyopathies] and medications used. As for the arrhythmia characteristics, they included arrhythmia duration and arrhythmia type. The authors used echocardiography as the imaging modality to determine extent of ventricular function recovery by comparing myocardial function before and after treatment of the culprit arrhythmia. The echocardiographic parameters that were assessed included LVEF, LV end-diastolic and end-systolic diameters, left atrial dimension, valvular abnormalities, right ventricular systolic pressures, and pulmonary arterial pressures.

In contrast to reported literature on the topic, Gopinathannair et al. found that none of the studied patient and arrhythmia characteristics had a significant effect on the recovery of ventricular function. Their results showed that initiation of aggressive arrhythmia treatment is warranted in patients with suspected AIC, regardless of arrhythmia duration, arrhythmia type, severity of baseline LVEF, and underlying structural heart disease. This was concluded based on the consistent substantial improvement in LVEF after arrhythmia suppression/elimination, mainly through rhythm control, across all different subgroups. In fact, the extent of LVEF improvement was similar whether comparing the group with known arrhythmia duration [KN] to that with unknown arrhythmia duration [UKN] $(21.2 \pm 9 \%$ vs $19.4 \pm 11 \%$, p-value $=0.16)$ or comparing the group with longest arrhythmia duration to the rest $(21.5 \pm 7.5 \%$ vs $21.0 \pm 9.2 \%$, p-value $=0.77)$. On the other hand, greatest improvement was seen in the group with lowest initial LVEF (24 \pm 17 vs $19 \pm 7 \%$; p-value $<0.0001$ ), making low index LVEF the only predictor of LVEF recovery after arrhythmia treatment in patients with AIC. However, the LVEF in these patients did not reach complete normalization; they had lower post-treatment LVEF compared to other groups ( $45 \pm 14$ vs $54 \pm 8 \%$; p $<0.0001$ ), a finding consistent with the available literature. Also similar to previous studies, the authors found that patients with PVCs experienced smaller extent of recovery compared to other arrhythmia types. The authors concluded by stressing the importance of suspecting AIC in patients having cardiomyopathy with a persistent arrhythmia and initiating aggressive arrhythmia treatment regardless of initial patient and arrhythmia characteristics.

As for the limitations of the study by Gopinathannair et al., there are few to mention. First, the study had a retrospective design and therefore findings only serve to generate hypotheses that need further testing and validation. Second, there is a lack of a control group to exclude interference of confounding factors. Although the use of Angiotensin-Converting Enzyme inhibitors (ACEi)/ Angiotension receptor blockers (ARB) did not independently predict LVEF improvement in multivariate analysis, it could still be a confounder given the lower rates of ACEi/ARB use in the cohort. Third, the timeframe of the study and the period of follow-up were not clearly defined. Fourth, there is lack of blinding of echocardiographic analyses which can potentially 
lead to inter- and intra-observer variability. Finally, the sample population was not diverse as it consisted in its majority of Caucasians.

The Gopinathannair et al. study demonstrated several points of strength. Among these are its multicenter nature and its relatively larger sample size compared to similar studies, giving its findings more weight. Moreover, the authors appropriately and clearly defined their inclusion and exclusion criteria. Furthermore, no funding was needed for the study which potentially frees it from direct or indirect influences on its design, execution and interpretation. Finally, the study has successfully improved our understanding of predictors of ventricular recovery in patients with AIC and showed that patients with AIC who had the longest duration of arrhythmia still had LV systolic function improvement with arrhythmia suppression/elimination. This study paves the way for prospective studies and randomized clinical trials to validate the generated hypotheses and corroborate the observational findings.

\section{References:}

1. AlJaroudi WA, Refaat MM, Habib RH, Al-Shaar L, Singh M, Gutmann R, Bloom HL, Dudley SC, Ellinor PT, Saba SF, Shalaby AA, Weiss R, McNamara DM, Halder I, London B; for the Genetic Risk Assessment of Defibrillator Events (GRADE) Investigators. Effect of Angiotensin Converting Enzyme Inhibitors and Receptor Blockers on Appropriate Implantable Cardiac Defibrillator Shock: Insights from the GRADE Multicenter Registry. Am J Cardiol Apr 2015; 115 (7): 115(7):924-31.

2. Refaat M, Mansour M, Singh JP, Ruskin JN, Heist EK: Electrocardiographic Characteristics in Right Ventricular Versus Biventricular Pacing in Patients With Paced Right Bundle Branch Block QRS Pattern. J Electrocardiol Mar-Apr 2011; 44 (2): 289-95.

3. Refaat MM, Hotait M, Tseng ZH: Utility of the Exercise Electrocardiogram Testing in Sudden Cardiac Death Risk Stratification. Ann Noninvasive Electrocardiol 2014; 19(4): 311-318.

4. Refaat MM, Hotait M, London B: Genetics of Sudden Cardiac Death. Curr Cardiol Rep Jul 2015; 17(7): 606.

5. Gopinathannair R, Etheridge SP, Marchlinski FE, Spinale FG, Lakkireddy D, Olshansky B. ArrhythmiaInduced Cardiomyopathies: Mechanisms, Recognition, and Management. J Am Coll Cardiol 2015; 66:1714-28.

6. Ehrlich JR, Nattel S, Hohnloser SH. Atrial fibrillation and congestive heart failure: specific considerations at the intersection of two common and important cardiac disease sets. J Cardiovasc Electrophysiol 2002; 13: 399-405.

7. Raymond-Paquin A, Nattel S, Wakili R, Tadros R. Mechanisms and Clinical Significance of ArrhythmiaInduced Cardiomyopathy. Can J Cardiol. 2018 Nov;34(11):1449-1460.

8. Ling LH, Kistler PM, Kalman JM, Schilling RJ, Hunter RJ. Comorbidity of atrial fibrillation and heart failure. Natl Rev Cardiol 2016; 13:131-47.

9. Nerheim P, Birger-Botkin S, Piracha L, Olshansky B. Heart failure and sudden death in patients with tachycardia-induced cardiomyopathy and recurrent tachycardia. Circulation 2004;110:247-52.

10. Ilkhanoff L, Gerstenfeld EP, Zado ES, Marchlinski FE. Changes in ventricular dimensions and function during recovery of atrial tachycardia-induced cardiomyopathy treated with catheter ablation. J Cardiovasc Electrophysiol 2007;18:1104-6. 